\title{
The Association of Lymphocyte count, CRP, D-Dimer, and LDH with Severe Coronavirus Disease 2019 (COVID-19): A Meta-Analysis
}

\author{
Almigdad H. M. Ali, Sagad Omer Obeid Mohamed, Ibrahim H. E. Elkhidir, \\ Mohamed Elata Hassan Elbathani, Abazr A. H. Ibrahim, Almutasim B. E. Elhas- \\ san, Mohammed Suliman Tawer Salman, Mazin A.M. Elhassan, Mahmoud \\ Elnil, and Abdelhamid Ibrahim Hassan Abuzied
}

Faculty of Medicine, University of Khartoum, Sudan

Corresponding Author:

Almigdad H. M. Ali;

Faculty of Medicine,

University of Khartoum, Qasr

Street. P.O. Box 11111

Khartoum, Sudan

Tel: 00249910174057

email:

Almigdad.h.m@gmail.com

Received 22 April 2020

Accepted 15 May 2020

Published 1 June 2020

Production and Hosting by

Knowledge E

(c) Almigdad H. M. Ali et al. This article is distributed under the terms of the Creative Commons

Attribution License, which permits unrestricted use and redistribution provided that the original author and source are credited.

Editor-in-Chief:

Prof. Mohammad A. M. Ibnouf

\section{Abstract}

Background: The rapid progression of Coronavirus disease 2019 (COVID-19) and its increasing burden on health systems necessitate the identification of parameters of severe infection to help in monitoring, prognoses and development of treatment algorithms.

Objectives: This review aims to investigate the association of lymphocyte count, CRP, $\mathrm{LDH}$, and D-Dimer with the severity of COVID-19.

Methods: This review was conducted according to the Preferred Reporting Items for Systematic Reviews and Meta-Analyses (PRISMA) guidelines. The databases of MEDLINE/PubMed, WHO-Virtual Health Library (VHL), and ScienceDirect were used for the systematic search. Random effects model was used to estimate the pooled standardized mean differences (SMD) with the corresponding 95\% confidence interval (Cl), using OpenMeta Analyst software.

Results: A total of 11 studies, with 2437 COVID-19 patients, which fulfilled the eligibility criteria were included in the meta-analysis. The analysis revealed that lymphocyte count was significantly lower in patients with the severe form of COVID-19 (SMD = - 1.025, $P$ value <.001). Also, the analysis of SMD showed that patients with severe COVID-19 have a significantly higher serum levels of CRP (SMD $=3.363, \mathrm{P}$ value $<.001$ ), D-Dimer (SMD = 1.073, $\mathrm{P}$ value <.001), and $\mathrm{LDH}(\mathrm{SMD}=3.345, \mathrm{P}$ value $<.001)$.

Conclusion: Low lymphocyte count and high levels of CRP, LDH, and D-Dimer are associated with severe COVID-19. These laboratory markers could be used as clinical indicators of worsening illness and poor prognosis of COVID-19.

Keywords: COVID-19, lymphocyte count, CRP, D-Dimer, LDH, Meta-analysis

\section{G OPEN ACCESS}

\section{Introduction}

In December 2019, Wuhan - the capital of Central China's Hubei province - witnessed the emergence of a new virus of the Coronaviridae family that caused several cases of severe respiratory disease [1, 2]. The virus was named by the World Health Organization 
(WHO) as a Severe Acute Respiratory Syndrome novel Coronavirus 2 (SARS-CoV-2) and the disease caused by this virus as Coronavirus Disease 2019 (COVID19) [1, 2]. The disease spread dramatically and reached other provinces in China and many other countries around the world, forcing the WHO to declare it as a global pandemic on March 11, 2020 [3]. Despite all the efforts made to contain the disease, it has spread to over 200 countries around the world, causing a significant burden to healthcare facilities, as well as economic burdens on international systems [4].

The SARS-CoV-2 is transmitted by infective respiratory droplets from infected patients, fomites, and surfaces reaching conjunctiva or respiratory mucosa. The incubation period usually ranges from 2 to 14 days after exposure, during which the patient may be infectious $[4,5]$. Animal-to-human transmission is still being investigated, knowing that similar coronaviruses are found in bats, rodents, and birds $[5,6]$.

Most people infected with COVID-19 experienced a mild-to-moderate respiratory illness, with cough and shortness of breath being the most common symptoms $[6,7]$. Approximately $14 \%$ of the patients developed a severe disease with complications like acute respiratory distress syndrome and respiratory failure, acute liver injury, acute kidney injury, cardiovascular complications, septic shock, and multi-organ failure [6, 7]. Older people and those with underlying co-morbidities like diabetes, chronic respiratory diseases, cardiovascular diseases, and immune-compromised patients had a higher risk of developing severe disease [8].

Patients with severe disease or complications require special care such as admission to intensive care units (ICU) with mechanical ventilation [9]. However, there is a wide gap between the total population and the number of intensive care beds available [9]. Further adding to the burden, ICU services cost the hospitals around 39\% of the total drug costs, $25 \%$ of the equipment, and $13 \%$ of the lab investigations [10]. Researchers are still working to find a definitive treatment of COVID-19, and there are ongoing trials on some antiviral, anti-inflammatories, and anti-malarial drugs such as hydroxychloroquine sulfate and chloroquine phosphate products to be used for certain hospitalized patients with COVD-19 [5, 11].

Diagnosis is dependent on the evaluation of the risk of contact with an infected patient, clinical signs and symptoms, and polymerase chain reaction testing of viral RNA on respiratory samples $[1,4,5]$. Other investigations have been used to evaluate the patient's condition and to predict severe outcomes of the disease such as complete blood count, D-Dimer, Procalcitonin, C-reactive protein (CRP), and lactate dehydrogenase $(\mathrm{LDH})[4,5]$. Two recent meta-analyses showed that increased procalcitonin values and thrombocytopenia are associated with a higher risk of severe COVID-19 [12, 13]. 
Other studies suggested that lymphocytes count, D-Dimer, CRP, and LDH have a role in the prognosis of COVID-19 [30, 31]. However, and to the best of our knowledge, there is no meta-analysis on these biomarkers. Therefore, the objective of this review is to assess the association between severe COVID-19 and these laboratory markers. Taking into consideration the disease burden and the limited resources and capacities of health facilities, it is very crucial to find parameters that support the clinical condition's risk assessment to aid the anticipation of severe complications.

\section{Materials and Methods}

\subsection{Search strategy and inclusion criteria}

In this meta-analysis, we followed the Preferred Reporting Items for Systematic Reviews and Meta-Analyses (PRISMA) statement [14]. The systematic literature search was performed using the electronic databases of Medline/PubMed, WHO-Virtual health library $(\mathrm{VHL})$, and ScienceDirect, without date or language restriction. The search terms used were "Novel coronavirus," "2019 nCoV," "COVID-19," "SARS-CoV-2," "Lymphopenia," "Lymphocytopenia," "leukopenia," "leukocytopenia," "D-Dimer," "C-reactive protein," "CRP," "lactate dehydrogenase," and "LDH" to ensure no possible relevant articles were missed. Also, we reviewed the articles referenced by the identified articles in this search.

All observational studies reporting sufficient information on lymphocyte count, CRP, LDH, and D-Dimer levels in both severe and non-severe COVID-19 patients, with a clear definition of severe illness, were included in the analysis for calculating the standardized mean difference (SMD) estimates. The exclusion criteria for the study were: case reports, editorials, letters, abstracts, and studies without sufficient data of interest. If two or more studies had the same patient population, the study with more data was included to avoid duplication.

The included studies determined severe COVID-19 based on the composite of respiratory distress with hypoxia and/or hypoxemia [15-17], the American Thoracic Society guidelines for community-acquired pneumonia [18], Youden's index [19], guidelines of expert Chinese group [20], non-survival [21], disease progression [22], Oxygen saturation < 90\% [23], the requirement of ICU admission [24], and the requirement of supplemental Oxygen [25].

The titles and abstracts of all articles retrieved from this search were screened for potential inclusion in this review. Then, potentially relevant studies were reviewed (full 
text) for inclusion according to the defined eligibility criteria, and data were extracted by independent reviewers using a data extraction form. The quality of the studies was assessed using the Newcastle - Ottawa scale. Any disparity among the reviewers at any step was resolved by discussion and consensus.

\subsection{Data analysis}

The statistical analyses were carried out using $\mathrm{R}$ language. The pooled SMD was calculated from the random-effects model due to the notable heterogeneity in this metaanalysis. Statistical heterogeneity was assessed with the $\mathrm{I}^{2}$ statistics and publication bias was determined through Egger's test and visual examination of the funnel plot [26].

\section{Results}

\subsection{Characteristics of the studies}

The schematic flow of the studies' identification and selection process is presented in Figure 1, and the summary of data from the included studies is shown in Supplementary Table 1. Our search retrieved records for 285 published articles, of which, full texts of 34 potentially relevant studies were retrieved for full-text screening and 23 studies were subsequently omitted because of duplication of the study populations and insufficient data to estimate the outcomes of interest. Last, a total of 11 studies with 2,437 patients, published from December 2019 to March 2020 were included for the analysis. All of them were from Mainland China [16-25], while one study was based in Singapore [26].

\subsection{Analysis of SMD of the lymphocyte count between the two groups of patients}

The pooled effect size showed that the lymphocyte count was significantly lower in patients with severe COVID-19 than patients with non-severe COVID-19 and the SMD = $-1.02(95 \% \mathrm{Cl},-1.33--0.74: \mathrm{P}<0.001$; Figure 2$)$. No evidence of publication bias was detected based on visual examination of the funnel plot and from the results of Egger's test $(p=0.59$; Figure $6 \mathrm{~A})$. 


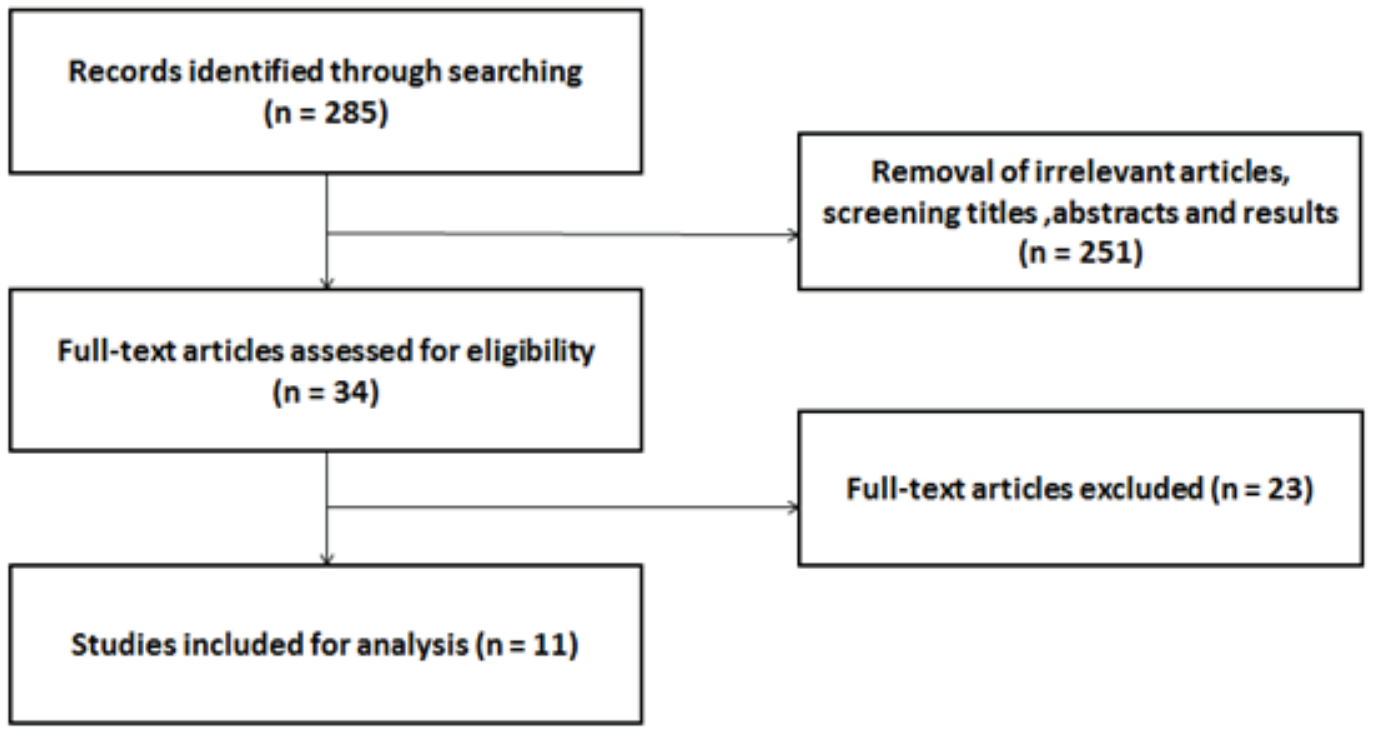

Figure 1: The flow diagram for the process of study selection and systematic review of literature.

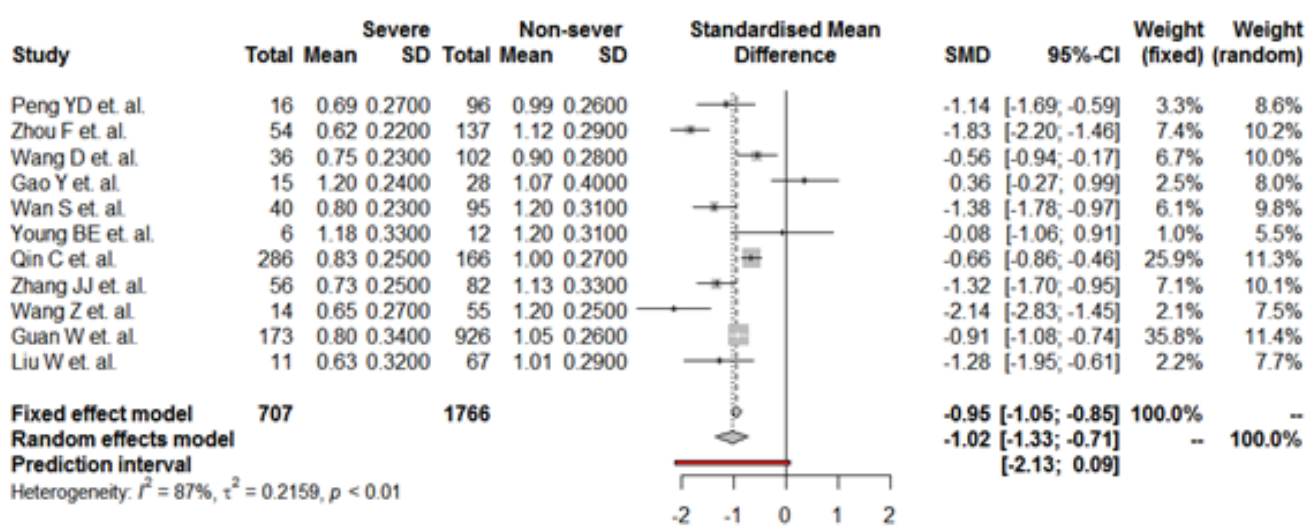

Figure 2: Pooled SMD of lymphocyte count estimates between the two groups of patients (severe and non-severe COVID-19).

\subsection{Analysis of SMD of the CRP between the two groups of patients}

The pooled effect size showed that CRP level was significantly higher in patients with severe COVID-19 than patients with non-severe COVID-19 with an SMD $=3.34(95 \% \mathrm{Cl}$, 2.19 - 4.49: $P<0.001$; Figure 3). A slight publication bias was detected based on visual examination of the funnel plot and from the results of Egger's test ( $p=0.03$; Figure 6B). The Duvall and Tweedie trim and fill method indicated that potential missing studies were three. 


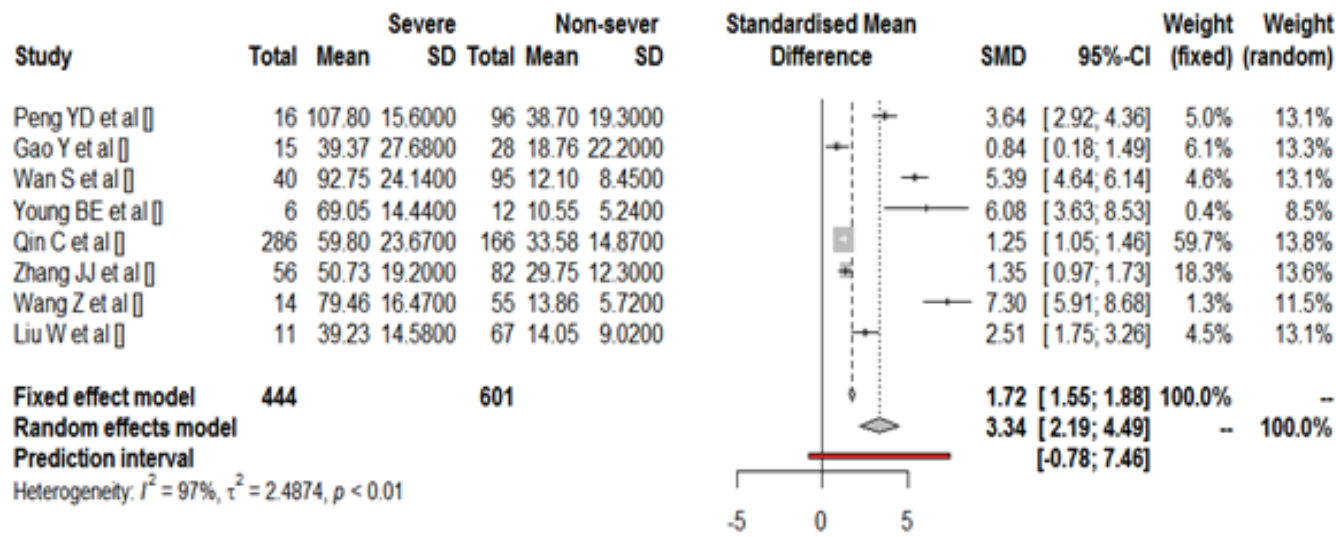

Figure 3: Pooled SMD of CRP estimates between the two groups of patients (severe and non-severe COVID-19).

\subsection{Analysis of SMD of the D-Dimer between the two groups of patients}

The pooled effect size showed that D-Dimer level was significantly higher in patients with severe COVID-19 than patients with non-severe COVID-19, with an SMD $=1.79$ (95\% $\mathrm{Cl}, 1.38$ - 2.19: $\mathrm{P}<0.001$; Figure 4). No evidence of publication bias was detected based on visual examination of the funnel plot and from the results of Egger's test $(p=0.97$ Figure 6C).

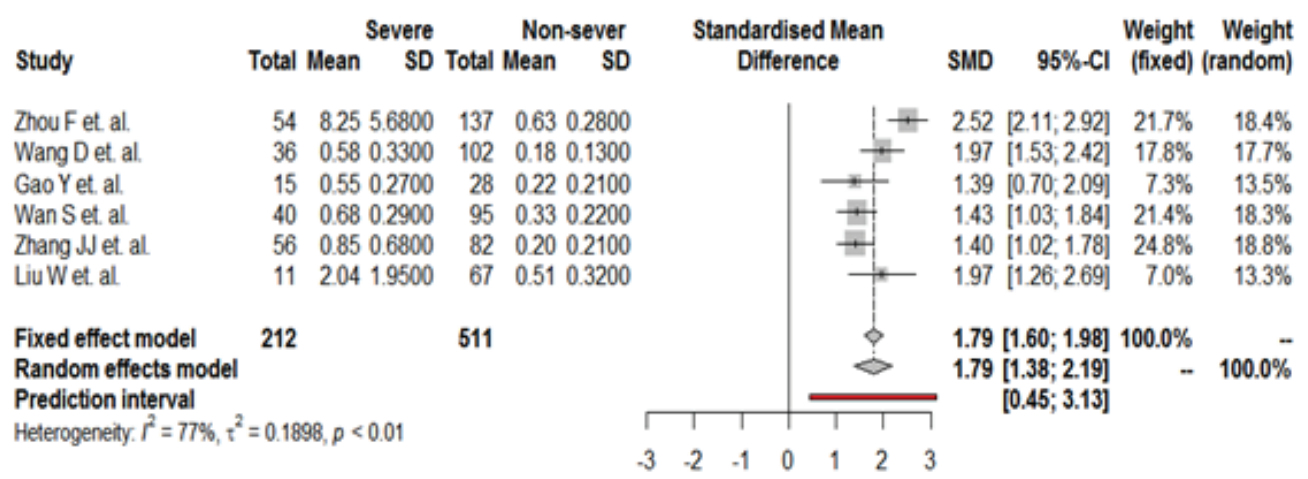

Figure 4: Pooled SMD of D-Dimer estimates between the two groups of patients (severe and non-severe COVID-19).

\subsection{Analysis of SMD of the LDH between the two groups of patients}

The pooled effect size showed that LDH level was significantly higher in patients with severe COVID-19 than patients with non-severe COVID-19, with an SMD $=3.34(95 \% \mathrm{Cl}$, 
1.92 - 4.77: $\mathrm{P}<0.001 ;$ Figure 5). No evidence of publication bias was detected based on visual examination of the funnel plot and from the results of the Egger's test $(p=$ 0.83; Figure 6D). The leave-one-out sensitivity analyses indicated that our results were robust and were not driven by any single study.

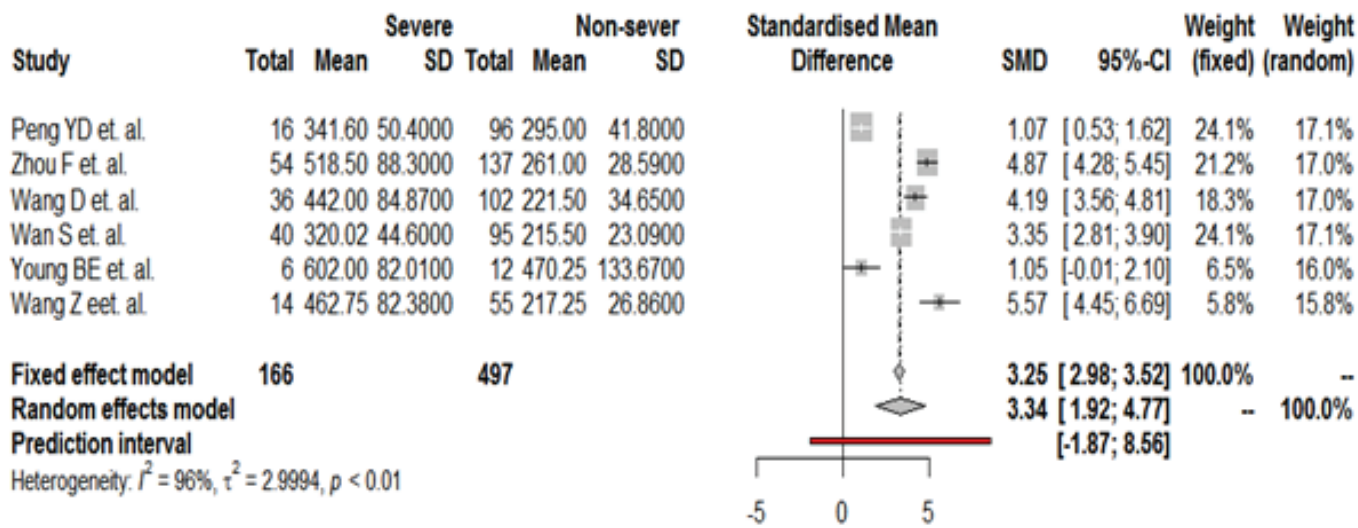

Figure 5: Pooled SMD of LDH estimates between the two groups of patients (severe and non-severe COVID-19).

A:

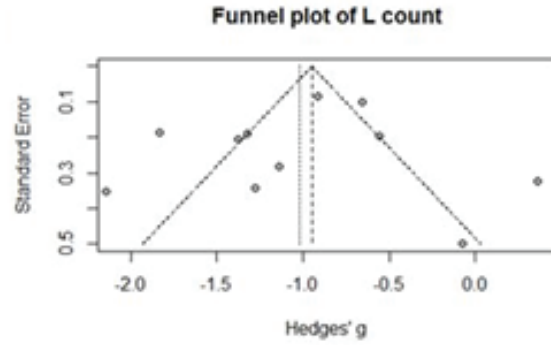

C:

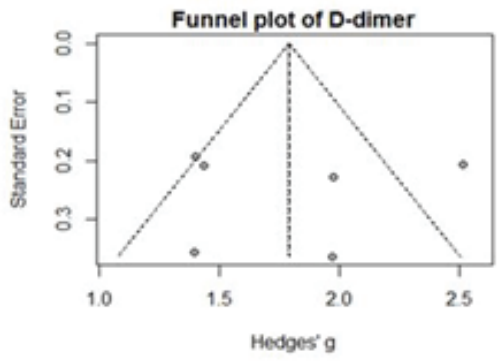

B:

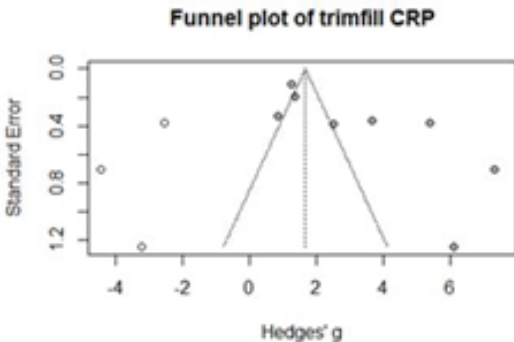

D:

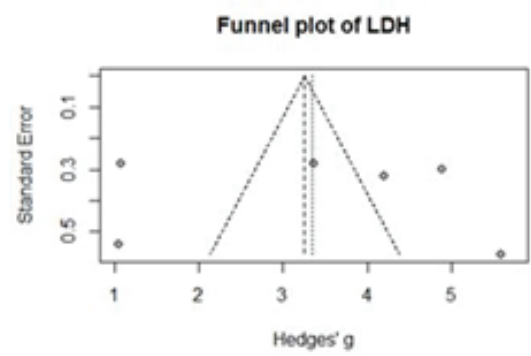

Figure 6: (A-D) Funnel plots for assessment of the publication bias.

\section{Discussion}

In addition to the clinical criteria, it is of great benefit to have laboratory markers for severe infection that will help in monitoring and prognosis and will be used to develop workup and treatment algorithms. This meta-analysis investigated the association between severe COVID-19 and several biomarkers. The analysis showed that 
patients with poor outcomes of COVID-19 are likely to have lymphopenia, as shown in Figure 2. Based on several studies, it has been suggested that the immune system is impaired during the disease and COVID-19 might damage T lymphocytes $[6,8,27]$. These studies showed a particular decrease in the CD4+ subset of lymphocytes and higher naive CD4+ cells than memory cells in severe cases $[6,8,27]$. The higher naive to memory cells ratio indicates that the immune system was impaired more severely $[6,8]$. The baseline lymphocyte count for these patients was not provided in these studies to determine if the lymphopenia was disease-related or was it present prior to the infection. If it was provided, these suggestions would have been more conclusive.

The changes in peripheral white blood cells were presumed to be caused by a cytokine storm in the body that generates a series of immune responses [27]. The levels of CRP and other inflammatory markers, such as IL-6, IL-8, IL-2R, and IL-10, were noticed to be higher in severe cases than non-severe cases [21]. Increased levels of cytokines, chemokines, and Neutrophils to Lymphocyte Ratio (NLR) in severe cases suggest a possible hyper-inflammatory response role in the pathogenesis of COVID-19 [20]. It is perplexing how elderly persons are more prone to severe COVID-19 despite their impaired immunity. This incongruence can be partially explained by the fact that elderly people have impaired innate and adaptive immunity. Innate immunity in healthy individuals manages to neutralize the virus early in the disease, keeping it from reaching the alveoli. The situation differs in elderly patients, where the innate immunity fails to do so, and the virus manages to reach the alveoli and replicate in high numbers. This triggers macrophages and lymphocytes to mount a vigorous response to eradicate virally infected cells. This response is associated with elevated levels of cytokines [28].

The D-Dimer level elevation in patients with severe disease may raise the suspicion of underlying abnormal blood coagulation function [20]. The effect of D-Dimer widens to include not only the correlation with the severity of the disease but also with mortality percentage, as shown by Zhou F. et al.'s study in Wuhan, China, who found that a DDimer level of $>1 \mathrm{mg} / \mathrm{ml}$ is associated with fatal outcome [21]. Gao Y. et al. showed that the clinical benefit of D-dimer level prediction of the severity of the disease will increase if it is combined along with IL6 level [20]. Also, the level of LDH, which is an inflammatory marker, shows a similar correlation with mortality percentages as that of D-dimer [19, 21]. LDH enzyme is found in the cytoplasm of all cells, thus any tissue damage would cause an increase in the serum level of the enzyme. Different tissues display different isoenzymes, but the isoenzymes were not specified in any of the reviewed studies. Therefore, the origin of the LDH couldn't be specifically identified [29]. 
The findings of this study need to be considered in the context of some limitations. All studies we found were conducted in Asia, whereas nowadays the majority of cases are in the United States and Europe. Due to the limitedness of the available data and variable definition of disease severity among the studies, there is a need for further studies to explore and clarify the prognostic role as well as the precise mechanisms underlying the changes in these biomarkers in patients with severe COVID-19.

\section{Conclusion}

Lymphopenia and high levels of CRP, LDH, and D-Dimer are associated with severe COVID-19. These laboratory markers could be used as clinical indicators of worsening illness and poor prognosis of COVID-19. This will help in developing different algorithms for managing COVID-19 patients according to the anticipated severity of the disease.

\section{Declarations}

\section{Ethical approval and consent to participate}

Not applicable

\section{Consent for publication}

Not applicable

\section{Availability of data and material}

The dataset generated during this study are available from the corresponding author on reasonable request.

\section{Competing interests}

None declared

\section{Funding}

No funding 


\section{Acknowledgment}

None to acknowledge

\section{Supplementary Materials}

Table 1 (Summary of the included studies in the review)

\section{Abbreviations}

- COVID-19: Coronavirus Disease 19

- SARS-Cov-2: Severe Acute Respiratory Syndrome novel Coronavirus 2

- CRP: C-Reactive Protein

- LDH: Lactate Dehydrogenase

\section{Authors Contribution}

AA conceptualized the research idea; $A A, S M$, and IE designed the study; $A A, S M, M E$, $\mathrm{MS}, \mathrm{Al}$, and $\mathrm{AE}$ undertook articles searching, articles assessment, and data extraction; SM and IE undertook data analysis. All authors interpreted the results and drafted the manuscript. All authors revised and approved the final manuscript and all of them agree to be accounted for the accuracy and integrity of all aspects of the work.

\section{References}

[1] Singhal, T. (2020). A review of Coronavirus Disease-2019 (COVID-19). The Indian Journal of Pediatrics, vol. 87, no. 4, pp. 281-286. Retrieved from: https://doi.org/10. 1007/s12098-020-03263-6

[2] WHO. (2020). Homepage. Retrieved from: https://www.who.int [accessed April 2, 2020]

[3] WHO. (2020). Coronavirus (COVID-19) outbreak. Retrieved from: https://www.who. int/westernpacific/emergencies/covid-19 [accessed April 2, 2020].

[4] WHO. (2020). Coronavirus (COVID-2019) situation reports. Retrieved from: https: //www.who.int/emergencies/diseases/novel-coronavirus-2019/situation-reports [accessed April 2, 2020]. 
[5] Centers for Disease Control and Prevention. (2020). Coronavirus disease 2019 (COVID-19). Retrieved from: https://www.cdc.gov/coronavirus/2019-ncov/hcp/ clinical-criteria.html [accessed April 2, 2020].

[6] Cascella, M., Rajnik, M., Cuomo, A., et al. (2020). Features, evaluation and treatment coronavirus (COVID-19) [Internet]. Treasure Island, FL: StatPearls Publishing. Retrieved from: http://www.ncbi.nlm.nih.gov/pubmed/32150360 [accessed April 3, 2020].

[7] BMJ Best Practice. (2020). Coronavirus disease 2019 (COVID-19) - Complications I BMJ Best Practice. Retrieved from: https://bestpractice.bmj.com/topics/en-us/ 3000168/complications [accessed April 2, 2020].

[8] Coronavirus Disease 2019 (COVID-19) [Internet]. Centers for Disease Control and Prevention. 2020 [cited 27 May 2020]. Retrieved from: https://www.cdc.gov/ coronavirus/2019-ncov/need-extra-precautions/people-at-higher-risk.html

[9] Rhodes, A., Ferdinande, P., Flaatten, H., et al. (2012). The variability of critical care bed numbers in Europe. Intensive Care Medicine, vol. 38, pp. 1647-1653. Retrieved from: https://link.springer.com/content/pdf/10.1007/s00134-012-2627-8.pdf

[10] Kılıç, M., Yüzkat, N., Soyalp, C., et al. (2019). Cost analysis on Intensive Care Unit costs based on the length of stay. Turkish Journal of Anaesthesiology \& Reanimation, vol. 47, no. 2, pp. 142-145. Retrieved from: https://pubmed.ncbi.nlm.nih.gov/31080956

[11] U.S. Food and Drug Administration. (2020). Coronavirus (COVID-19) update: daily roundup March 30, 2020. Retrieved from: http://www.fda.gov/news-events/pressannouncements/coronavirus-covid-19-update-daily-roundup-march-30-2020 [accessed April 2, 2020].

[12] Lippi, G., Plebani, M., and Michael Henry, B. (2020). Thrombocytopenia is associated with severe coronavirus disease 2019 (COVID-19) infections: a meta-analysis. Clinica Chimica Acta, vol. 506, pp. 145-148. Retrieved from: https://doi.org/10.1016/j.cca. 2020.03 .022

[13] Lippi, G. and Plebani M. (2020). Procalcitonin in patients with severe coronavirus disease 2019 (COVID-19): a meta-analysis. Clinica Chimica Acta, vol. 505, pp. 190191. Retrieved from: https://doi.org/10.1016/j.cca.2020.03.004

[14] Liberati, A., Altman, D. G., Tetzlaf, J., et al. (2009). The PRISMA statement for reporting systematic reviews and meta-analyses of studies that evaluate health care interventions: explanation and elaboration. Journal of Clinical Epidemiology, vol. 62, no. 10, pp. e1-34.

[15] Yudong, P., Kai, M., Hongquan, G., et al. (2020). Clinical characteristics and outcomes of 112 patients with cardiovascular disease infected with novel coronavirus 
pneumonia. Chinese Journal of Cardiovascular Diseases, vol. 48, p. E004. DOI:10.3760/cma.j.cn112148-20200220-00105.

[16] Wan S, Xiang Y, Fang W, Zheng Y, Li B, Hu Y, Lang C, Huang D, Sun Q, Xiong Y, Huang $X$. Clinical features and treatment of COVID-19 patients in northeast Chongqing. Journal of medical virology. 2020 Mar 21. Retrieved from: https://onlinelibrary.wiley. com/doi/abs/10.1002/jmv.25783

[17] Zhang JJ, Dong X, Cao YY, Yuan YD, Yang YB, Yan YQ, Akdis CA, Gao YD. Clinical characteristics of 140 patients infected with SARS-CoV-2 in Wuhan, China. Allergy. 2020 Feb 19. Retrieved from: https://onlinelibrary.wiley.com/doi/full/10.1111/all.14238

[18] Guan, W., Ni, Z., Hu, Yu., et al. (). Clinical Characteristics of Coronavirus Disease 2019 in China. The New England Journal of Medicine, vol 382, pp. 1708-1720. DOI:10.1056/NEJMoa2002032

[19] Gao Y, Li T, Han M, Li X, Wu D, Xu Y, Zhu Y, Liu Y, Wang X, Wang L. Diagnostic utility of clinical laboratory data determinations for patients with the severe COVID19. Journal of medical virology. 2020 Mar 17. Retrieved from: https://onlinelibrary. wiley.com/doi/full/10.1002/jmv.25770

[20] Qin, C., Zhou, L., Hu, Z., et al. (2020). Dysregulation of immune response in patients with COVID-19 in Wuhan, China. SSRN Electronic Journal. Retrieved from: https: //dx.doi.org/10.2139/ssrn.3541136

[21] Zhou, F., Yu, T., Du, R., et al. (2020). Clinical course and risk factors for mortality of adult inpatients with COVID-19 in Wuhan, China: a retrospective cohort study. The Lancet, vol. 395, no. 10229, pp. 1054-1062.

[22] Liu, W., Tao, Z., Lei, W., et al. (2020). Analysis of factors associated with disease outcomes in hospitalized patients with 2019 novel coronavirus disease. Chinese Medical Journal. DOI:10.1097/CM9.0000000000000775

[23] Wang, Z., Yang, B., Li, Q., et al. (2020). Clinical features of 69 cases with coronavirus disease 2019 in Wuhan, China. Clinical Infectious Diseases [Online ahead of print]. Retrieved from: https://pubmed.ncbi.nlm.nih.gov/32176772/

[24] Wang, D., Hu, B., Hu, C., et al. (2020). Clinical characteristics of 138 hospitalized patients with 2019 novel Coronavirus-infected pneumonia in Wuhan, China. JAMA, vol. 323, no. 11, pp. 1061-1069. DOI:10.1001/jama.2020.1585

[25] Young, B. E., Ong, S. W. X., Kalimuddin, S., et al. (2020). Epidemiologic features and clinical course of patients infected with SARS-CoV-2 in Singapore. JAMA. DOI:10.1001/jama.2020.3204 
[26] Borenstein, M., Hedges, L. V., Higgins, J., et al. (2010). A basic introduction to fixedeffect and random-effects models for meta-analysis. Research Synthesis Methods. Vol. 1, no. 2, pp. 97-111.

[27] Rodriguez-Morales, A. J., Cardona-Ospina, J. A., Gutiérrez-Ocampo, E., et al. (2020). A systematic review and meta-analysis. Travel Medicine and Infectious Disease, vol. 34, p. 101623. Retrieved from: https://doi.org/10.1016/j.tmaid.2020.101623.

[28] Abdulamir, A. S. and Hafidh, R. R. (2020). The possible immunological pathways for the variable immunopathogenesis of COVID-19 infections among healthy adults, elderly and children. Electronic Journal of General Medicine, vol. 17, no. 4.

[29] Burtis, C. A., Ashwood, E. R., and Bruns, D. E. (Ed.) (2006). Tietz Textbook of Clinical Chemistry and Molecular Diagnostics, pp. 601-602. St. Louis, MO: Elsevier Saunders.

[30] Harenberg J, Favaloro E. COVID-19: progression of disease and intravascular coagulation - present status and future perspectives. Clinical Chemistry and Laboratory Medicine (CCLM). 2020;0(0). Retrieved from: https://www.degruyter. com/view/journals/cclm/ahead-of-print/article-10.1515-cclm-2020-0502/article-10. 1515-cclm-2020-0502.xml

[31] Sun Y, Dong Y, Wang L, Xie H, Li B, Chang C, Wang FS. Characteristics and prognostic factors of disease severity in patients with COVID-19: The Beijing experience. Journal of Autoimmunity. 2020 Apr 24:102473. Retrieved from: https://www.ncbi.nlm.nih.gov/ pmc/articles/PMC7180376/ 